\title{
Orbital Prosthesis: A Novel Treatment Approach
}

\author{
CK Anulekha Avinash, Ramesh Nadiger, Sathyabodh S Guttal, K Lekha
}

\begin{abstract}
The disfigurement associated with loss of eye can cause significant physical and emotional disturbance. Rehabilitation of facial defects is a complex task, requiring an individualized design of the technique for each patient and also a technique sensitive procedure, visual assessment alone may not be accurate. We report a case of 23-year-old male patient with rhabdomyosarcoma of the eye, which was treated with chemotherapy and surgical enucleation, and was rehabilitated with silicon orbital prosthesis by constructing a custom ocular prosthesis to achieve ideal fit and esthetics.
\end{abstract}

Keywords: Orbital prosthesis, Phthisical eye, Prosthetic rehabilitation.

How to cite this article: Avinash CKA, Nadiger R, G uttal SS, Lekha K. Orbital Prosthesis: A Novel Treatment Approach. Int J Prosthodont Restor Dent 2012;2(1):19-23.

\section{Source of support $\mathrm{Nil}$}

\section{Conflict of interest: None}

\section{INTRODUCTION}

Orbital rehabilitation is complex process which requires specificity in the technique according to the particular patient. Orbital prosthesis is done after enucleation of the eye due to trauma, tumor, infection malignancy or congenital abnormality. Prosthetic rehabilitation is the treatment of choice when surgical reconstructive procedure does not show satisfactory results. Loss of eye is emotional and physical problem to the patient, so lesions involving the facial defects require prosthetic rehabilitation. An orbital prosthesis is created to restore a more normal anatomical structure and cosmetic defect created by these conditions in a person.

Surgical procedures adopted for the removal of an eye are classified by Peyman, Saunders and Goldberg into three general categories: Enucleation, evisceration and exenteration. Enucleation is a surgical procedure in which the globe and the attached portion of the optic nerve are excised from the orbit. Evisceration is removal of the contents of globe while leaving the sclera and extraocular muscles intact. Exenteration is the most radical of the three procedures and involves removal of the eye, adnexa, and the part of the bony orbit. ${ }^{1}$ Prosthesis for orbital defects are made from a variety of materials, such as polymethyl methacrylate, polyurethane elastomer, silicone elastomer or urethane backed medical grade silicone. They are mainly retained using mechamical means of anatomical undercuts, spectacle frames or by the use of osseointegrated extraoral implants. ${ }^{2,3}$ Present paper highlights a simple and economical technique to rehabilitate a patient with orbital prosthesis using medical grade silicone material.

The earliest known examples of restorations dates to the fourth dynasty in Egypt; excavations of tombs have provided evidence of eye replacement by using precious stones, earthenware, copper, gold, enamelled bronze in the shrunken socket. U ntil W orld W ar II, the glass eye was the most popular prosthetic eye manufactured. The glass eye was, however, difficult to manufacture and hazardous when it exploded. $O$ ne of the pioneers to use glass eye was A mbroise Pare (1510-1590). In 1944, M urphy and N irroren fabricated physiologic ocular prosthesis in the U nited States Navy Dental Corps. ${ }^{4}$ Pare al so used glass and porcelain for eyes, which was a great step forward. Later on methyl methacrylate prosthesis became popular since they offered superior strength, as well as the shape and size could be modified. ${ }^{5}$ Recently, flexible maxillofacial material, such as silicone is the material of choice, for having lifelike appearance.

\section{CASE REPORT}

A 45-year-old male was referred to the Department of Prosthodontics for evaluation and treatment of a patient who was suffereing from facial disfigurement with loss of his left eye. History of the patient reveal ed, exenteration of orbit, which had been carried out 1 year before due to eradication of rhabdomyosarcoma. Extraoral examination of the patient showed a large orbital defect on right side (Fig. 1). Extraorally, no definite bony or soft tissue undercut was

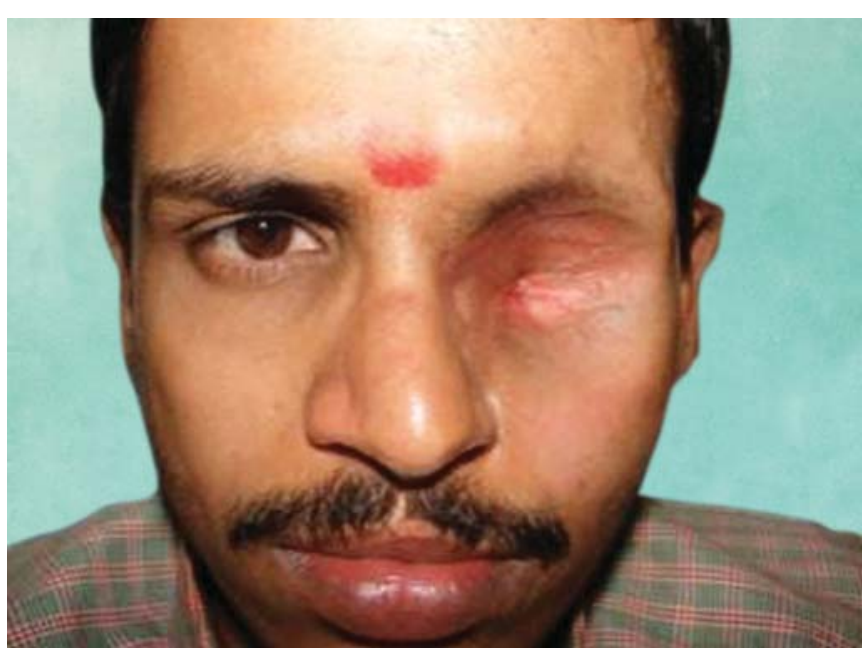

Fig. 1: Pretreatment photograph 
found to help in the retention of the prosthesis. A spectacle retained silicone orbital prosthesis was planned for the patient to make him socially presentable for his remaining days.

\section{PROCEDURE}

A fter evaluation and inspection of the defect, the diameter of the iris and pupil on the intact side was measured using callipers. A rea of impression was lubricated and impression was done with irreversible hydrocolloid (alginate). Wet gauze was spread over the hydrocolloid before it was set and the entire impression was reinforced with quick setting dental plaster (Figs 2A to C). Impression was examined for completeness after removal and subsequently, a cast was poured in dental stone type II. The master cast was duplicated on which modeling wax was adapted to fabricate shim (Fig. 3). This waxed up shim was processed using heat polymerizing clear acrylic resin (Fig. 4).

The resin base was retrieved, finished and polished. The fit of the base was checked on the cast.

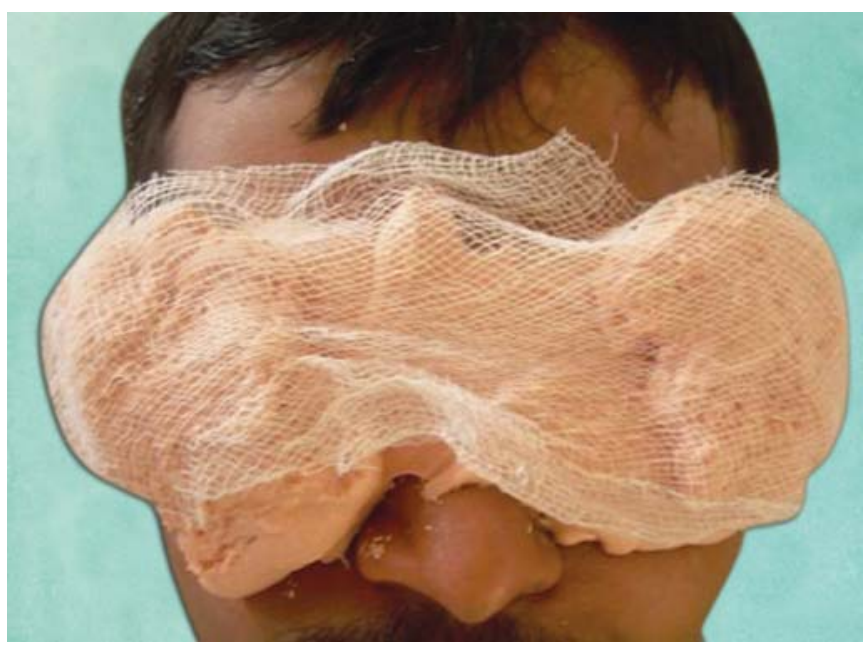

Fig. 2A: Direct impression with alginate

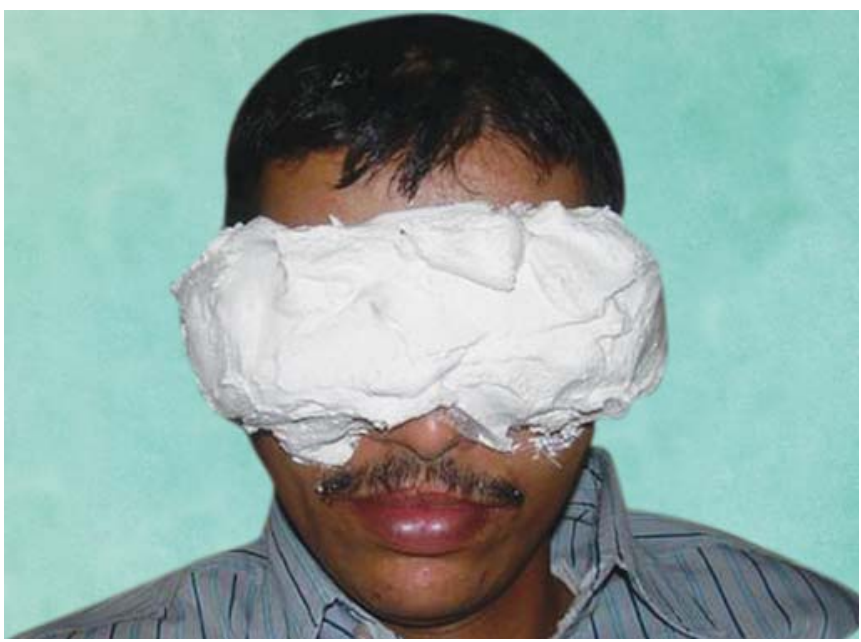

Fig. 2B: Reinforcement of alginate by dental plaster

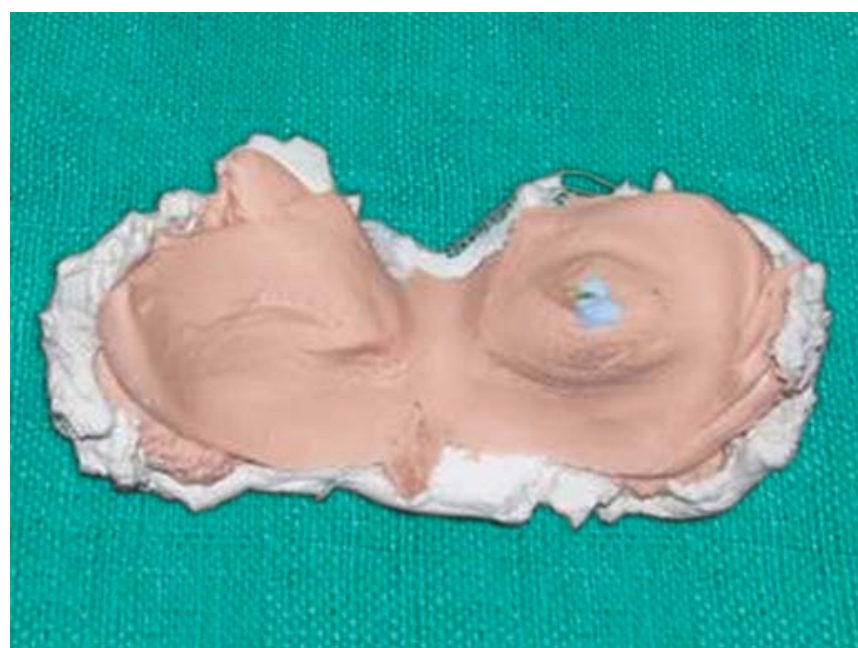

Fig. $\mathbf{2 C}$ : Impression after retrieval

The shim provided the attachment of the silicone prosthesis to the frame of the eye wear. The acrylic shim not only formed a steady base but also aided in providing mechanical retention for the silicone orbital prosthesis. $M$ easurements were made from the patient's facial midline

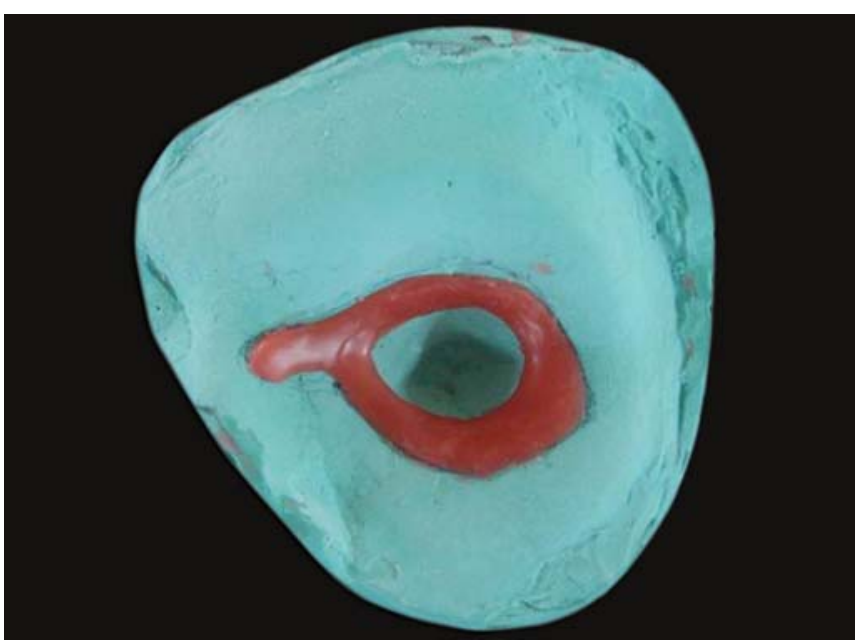

Fig. 3: Wax pattern for the fabrication of acrylic shim

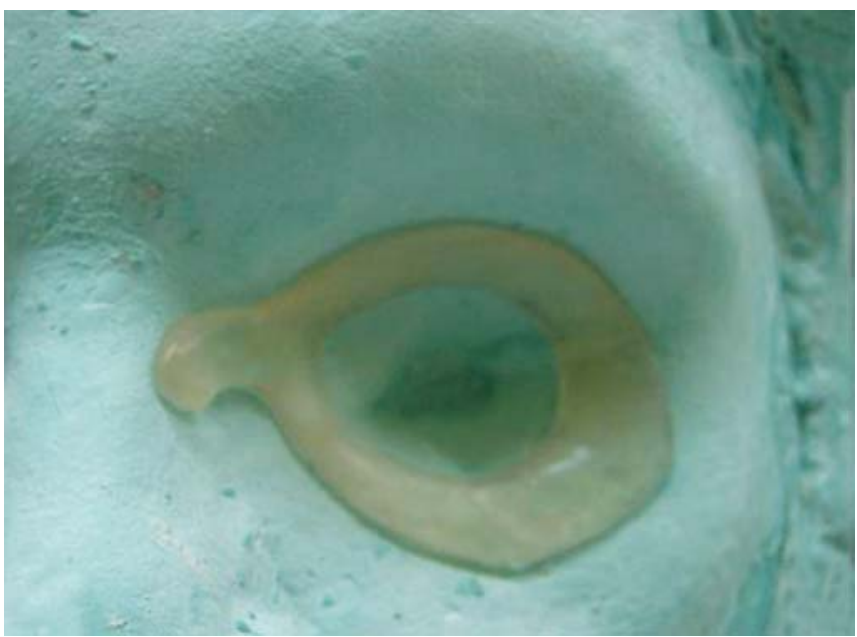

Fig. 4: Heat polymerizing clear acrylic shim on the master cast 
to the center of the pupil to the facial midline and from the inner canthus of the eye to the nasal bridge. Both the measurements were made when the patient was asked to look and fix the contralateral eye at distant gaze (Fig. 5). These measurements were transferred on the cast to help in the position of the ocular portion of the orbital prosthesis.

A suitable stock ocular prosthesis was selected that closely matched the color, size and shape of the iris and sclera of the other eye. The ocular portion needed minor alterations to make it fit into the socket as the defect was relatively large. The acrylic shim was exposed only at the bridge of the nose for attachment to the eyeglass frame. The eye was then secured in position on a bed of modeling wax according to the position gained using the measurements of the other eye. The anteroposterior position was adjusted and verified on the patient when observed from profile and from the top of the head. Once the position was confirmed, the eyelids and the remaining portion was sculpted in wax (Fig. 6) and tried in the patient's orbital defect. Consent from the patient regarding the appearance

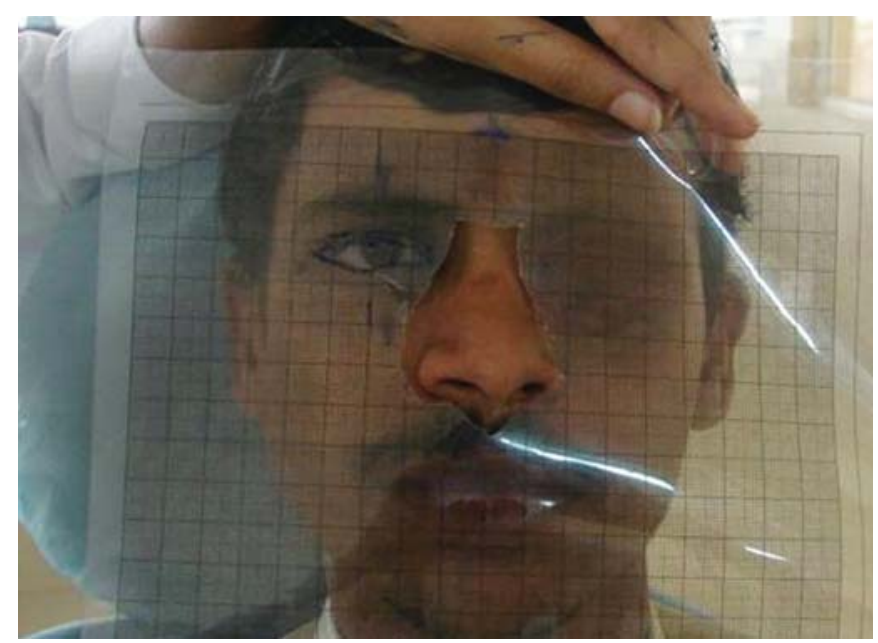

Fig. 5: Grid in place

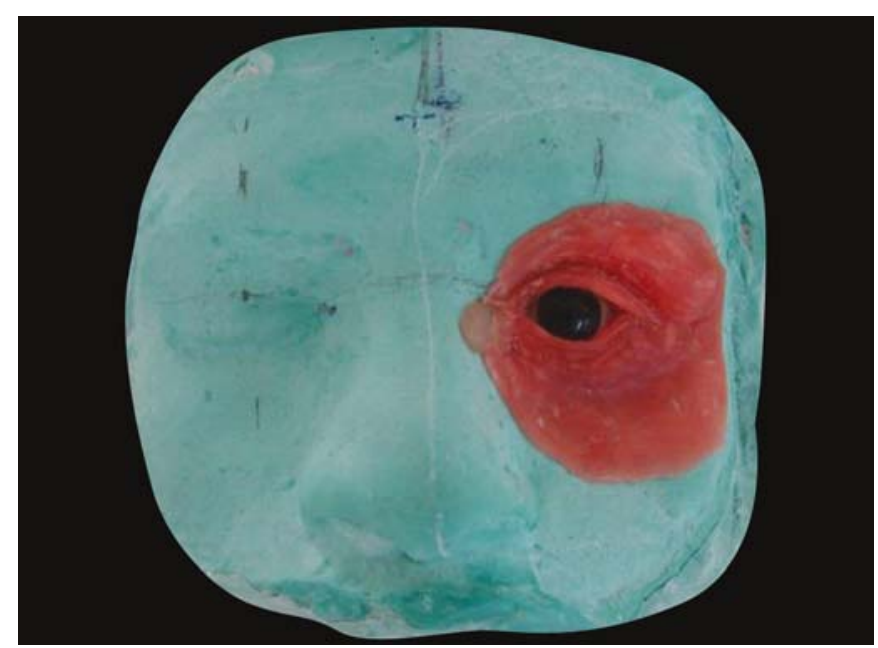

Fig. 6: Sculpted wax pattern of the prosthesis was obtained. The wax sculpted prosthesis with the duplicated cast was flasked and dewaxed. Room temperature vulcanizing (RTV) medical-graded silicone material (Molloplast) was mixed according to manufacturer's instructions. Pigment stains were blended into the base color of silicon for intrinsic staining at the time of mix to gain the approximate skin shade of the patient. A fter a close shade match with the patient, medical-graded silicone was packed into the mould and was left to cure at room temperature. Following polymerization the prosthesis was deflasked, retrieved and finished. Natural hair was stitched over eyebrow area and upper, lower eyelids of the silicone prosthesis using 23 gauge syringe needle (Fig. 7). The eyeglass frame was selected and tried on the patient. With both the eyeglass frame and prosthesis placed in situ, the glass frame was attached to the acrylic shim with the help of cyanoacrylate resin adhesive. The attachment was reinforced with clear autopolymerizing acrylic resin. Thus the silicone orbital prosthesis retained by the eyeglass frame and the acrylic shim (Fig. 8). Finally home care instructions

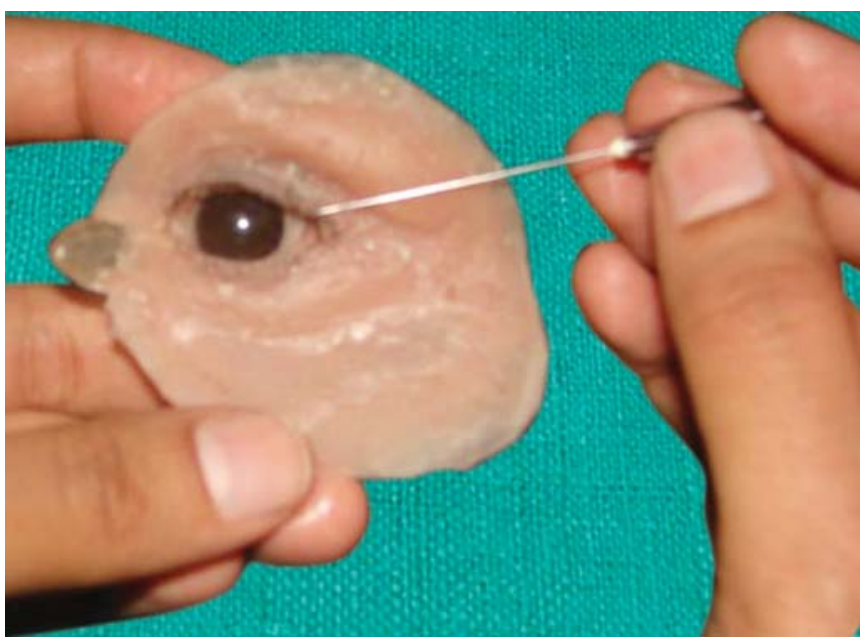

Fig. 7: Stitching of natural hair to the silicone orbital prosthesis

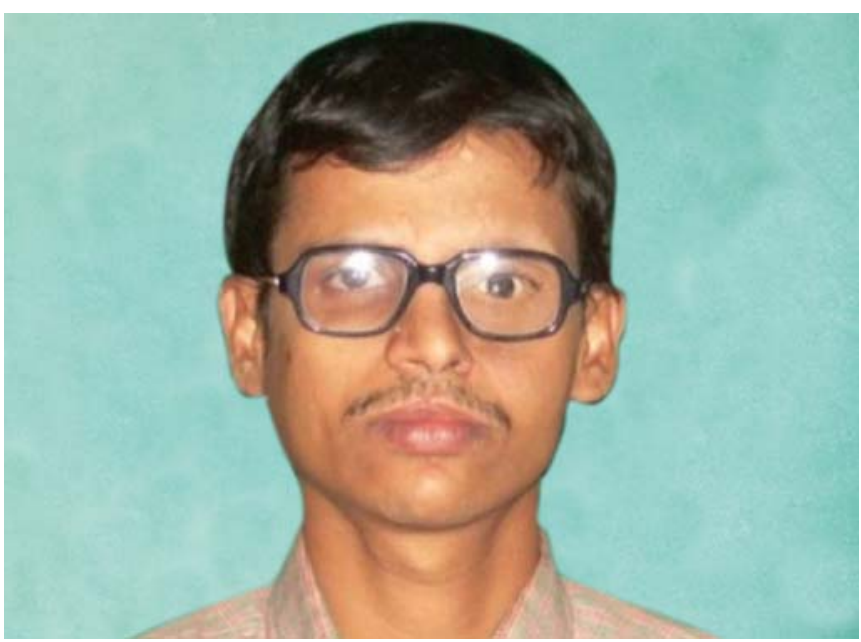

Fig. 8: Post-treatment photograph along with silicone artificial eye 
were given, and frequent follow-up was carried out for the evaluation of function of prosthesis. The patient was satisfied with the function and esthetics provided by the prosthesis, as it made him socially presentable for his remaining days.

\section{DISCUSSION}

Nowadays orbital reconstruction by plastic surgery or the use of osseointegrated implants are in use. If the patient does not desires or if it is not possible for the patient to get such sophisticated treatment modalities, a custom-made silicon orbital prosthesis is always a good option. A fter exenteration of the orbit and its contents, psychological and cosmetic rehabilitation should be planned subsequently which will be the atmost concern. O rbital prosthesis presents as simple, attractive and viable alternative when esthetic and functional needs are beyond the reach of local reconstructive procedures. Present case came with an urge for cosmetic as well a social concern which was successfully treated by silicon orbital prosthesis.

Selection of a reasonable maxill ofacial prosthetic material and economically feasible retentive aid should be the goal of rehabilitating such patients. Since silicone has better marginal adaptation and lifelike appearance, it has been used for the fabrication of orbital prostheses. Silicones have been used for over 50 years in the field of maxillofacial prosthetics, with desirable material properties including flexibility, biocompatibility, ability to accept intrinsic and extrinsic colorants, chemical and physical inertness and mouldability. ${ }^{6}$

The most commonly used conventional method to retain orbital prostheses is the eyeglass frames and anatomic retentive undercuts. ${ }^{7}$ The patient treated in this report had no anatomical undercuts at the defect; hence, an eyeglass frame was the only alternative to retain the silicone orbital prosthesis. As silicone prosthesis does not directly bond to the eyeglass. So, an acrylic shim was fabricated, which not only formed a steady base for the orbital prosthesis but al so aided in the attachment of the silicone artificial eye to the eyew ear.

The most commonly used material for sculpting the patterns are the modeling clay and waxes. However, many individuals who once preferred sculpting clay have turned to use wax because of the possibility of residual oils from the clay contaminating the mold surfaces, which can interfere with the platinum catalyst employed in modern silicon prosthesis material. ${ }^{8}$ The material used to sculpt the orbital wax pattern was modeling wax, as medical grade silicone was used in the fabrication of artificial eye. The silicone eye prosthesis provided all the advantages of being light weight, better esthetics with lifelike appearance than acrylic prosthesis. The thin flash of the silicone eye merged with the adjacent skin to give a lifelike appearance. Use of adhesive was not required as the prosthesis was attached eyew ear. So, the chances of allergic reaction caused due to the use of adhesives or silicone were also overcomed, as in this case, more of tissue contact was with acrylic shim. Further, instead of sticking artificial eye lashes, natural hair was stitched over eyebrow area and upper, lower eyelids of the silicone prosthesis using syringe needle. This enhanced the natural touch to the artificial eye.

Deep orbital deformity with devastating cosmetic and functional implications that require expensive and technically challenging oculoplastic intervention, are al ways associated with psychological effects. Orbital prosthesis presents an attractive and viable al ternative when esthetic and functional demands are beyond the capacity of local reconstructive efforts. ${ }^{9}$

A blative surgical procedure incurs major financial burden, and hence, the patient may seek a prosthetic treatment that is economical. Therefore, selection of a reasonable maxillofacial prosthetic material and economically feasible retentive aid should be the goal of rehabilitating such patients. ${ }^{10}$ Since, silicone has better marginal adaptation and lifelike appearance, it has been used for the fabrication of orbital prostheses.

\section{CONCLUSION}

The optimum cosmetic and functional results of a custommade prosthesis enhance the patient rehabilitation to a normal lifestyle. This paper elaborates the technique of fabrication of custom-made orbital prosthesis for a patient whose eye was exenterated as a result of rhabdomyosarcoma of the left eye.

It is the birthright of every human to appear socially acceptable. So, as prosthodontist, it is our duty to implement our knowledge into practicality and fabricate the prosthesis in an acceptable fashion to meet the physiologic, anatomic, functional and cosmetic requirements of the patient. In this way, we can help patient begin to heal medically and emotionally as soon as possible.

\section{ACKNOWLEDGMENT}

The authors thank their teacher, late Dr N P Patil, for his encouragement in academic and teaching.

\section{REFERENCES}

1. M ishra SK, Ramesh C. Reproduction of custom-made eye prosthesis manoeuvre: A case report. J Dent Oral Hyg 2009;1: 59-63. 
2. Wolfaardt J, Gehl G, Farmand M, Wilkes G. Indications and methods of care for aspects of extraoral osseointegration. Int J Oral M axfac Surg 2003;32:124-31.

3. Beumer J, Ma T, M arunick M. Restoration of facial defects: Etiology, disability and rehabilitation. In: Beumer J, Curtis A, $M$ arunick MT (Eds). M axillofacial rehabilitation: Prosthodontic and surgical considerations. St L ouis: Ishiyaky Euro A merica 1996; 377-453.

4. Beumer J, Curtis TA, M arunick M T. M axillofacial rehabilitation: Prosthodontic and surgical considerations (2nd ed). St Louis: Ishiyaku E uroamerica 1996;431-49.

5. Supriya M. Prosthodontic rehabilitation of a patient with an orbital defect using simplified approach. J Ind Prosth Soc 2008;8(2):16-18.

6. Jani RM, Schaaf NG. An evaluation of facial prostheses. J Prosthet Dent 1978;39:546-50.

7. Parel SM . Diminishing dependence on adhesives for retention of facial prosthesis. J Prosthet Dent 1980;43:552-60.

8. Taylor DT. Clinical Maxillofacial Prosthetics. Quintessence Publishing Co, Inc 2000: pg 99.

9. Konstantinidis L, Scolozzi P, Hamedani M. Rehabilitation of orbital cavity after total orbital exenteration using oculofacial prostheses anchored by osseointegrated dental implants posed as a one step surgical procedure. Klin Monbl Augenheilkd 2006;223:400-04.
10. Rodrigues S, Shenoy VK, Shenoy K. Prosthetic rehabilitation of a patient after partial rhinectomy: A clinical report. J Prosthet Dent 2005;93:125-28.

\section{ABOUT THE AUTHORS}

\section{CK Anulekha Avinash (Corresponding Author)}

A ssistant Professor, D epartment of Prosthodontics, Kamineni Institute of Dental Sciences, Nalgonda, Andhra Pradesh, India, Phone: 08121319805, e-mail: anulekhaavinash@ gmail.com

\section{Ramesh Nadiger}

Professor and Head, Department of Prosthodontics, SD M College of Dental Sciences, D harwad, Karnataka, India

\section{Sathyabodh S Guttal}

Professor, Department of Prosthodontics, SDM College of Dental Sciences, D harwad, Karnataka, India

\section{K Lekha}

Professor, Department of Prosthodontics, SDM College of Dental Sciences, D harwad, Karnataka, India 